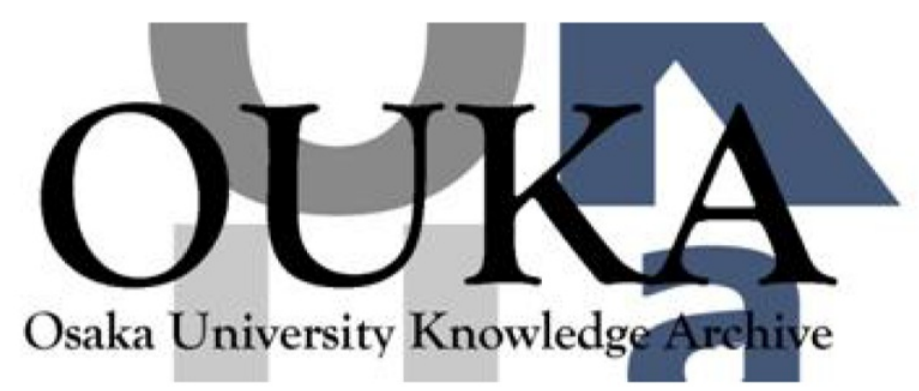

\begin{tabular}{|c|l|}
\hline Title & $\begin{array}{l}\text { Polarization force on a charged particulate in } \\
\text { a nonuniform plasma }\end{array}$ \\
\hline Author(s) & Hamaguchi, S.; Farouki, R. T. \\
\hline Citation & Physical Review E. 49(5) p.4430-p.4441 \\
\hline Issue Date & $1994-05$ \\
\hline oaire:version & VoR \\
\hline URL & https://hdl.handle.net/11094/78507 \\
\hline rights & $\begin{array}{l}\text { Copyright (1994) by the American Physical } \\
\text { Society }\end{array}$ \\
\hline Note & \\
\hline
\end{tabular}

Osaka University Knowledge Archive : OUKA

https://ir. Library. osaka-u. ac. jp/

Osaka University 


\title{
Polarization force on a charged particulate in a nonuniform plasma
}

\author{
S. Hamaguchi and R. T. Farouki \\ IBM Thomas J. Watson Research Center, P.O. Box 218, Yorktown Heights, New York 10598
}

(Received 6 December 1993)

\begin{abstract}
The force on a charged particulate or "dust grain" in a plasma is derived in the presence of a density gradient and an externally applied electric field. The Debye sheath around the particulate is usually deformed (i.e., polarized) under such conditions, and exerts a force on the particulate. This sheathpolarization force is always in the direction of decreasing Debye length, regardless of the sign of the particulate charge. The magnitude of the polarization force is estimated under typical glow discharge conditions.
\end{abstract}

PACS number(s): 52.25.Fi, 52.25.Vy

\section{INTRODUCTION}

Small charged dust grains or "particulates" are observed in a wide variety of plasma environments, ranging from low-pressure discharges used in industrial applications to the interstellar medium. As plasmas have been used increasingly in industrial material processing, the presence of particulate contamination has been recognized to pose serious problems for product yield and reliability $[1-5]$.

If the particulate density is sufficiently high, the interparticle potential energies may substantially exceed their thermal kinetic energies [6], and the particulate system then exhibits behavior similar to a liquid [7] or classical one-component plasma [8-11]. In isolation, an electrically charged grain in a nonuniform plasma interacts with its own sheath field, any externally applied electric field, and plasma flows. Interactions with other particulates and the background plasma determine the transport properties of the particulate system in a nonuniform plasma.

We are concerned here with the transport of an isolated particulate, in particular with the force exerted on it by the ambient plasma. Since the sizes of particulates and their Debye sheaths are typically small on the macroscopic scale (e.g., the dimensions of the discharge chamber), the particulate dynamics are determined by local plasma parameters such as the electric field, plasma density gradient, etc., at the position of the particulate. We will exploit this scale discrepancy to obtain the force as a function of macroscopic plasma parameters. Such a functional dependence may be used in conjunction with macroscopic (e.g., fluid or particle in cell) simulations to determine the overall particulate transport characteristics in the discharge.

As is well known, a charged particle in a plasma is surrounded by a Debye sheath, which shields its long-range Coulomb field. Particulates in plasmas are typically negatively charged - due to the high mobility of the plasma electrons - so their Debye sheaths comprise an excess of positive ions and a deficiency of electrons. The problem addressed in this paper is that of whether, in the presence of an external electric field, the Debye shielding

effectively diminishes the magnitude of this field at the particle location (by, for example, a redistribution of the sheath charges).

As will be clarified below, the Debye sheath does modi$f y$ the externally applied field by a redistribution (i.e., polarization) of the sheath charges. However, the external field is not necessarily "shielded" or diminished at the position of the particulate: the Debye sheath may either reduce or enhance the external field, depending on the sense of the mean density gradient at the particulate. In a nonuniform plasma, the deviation of the force from the nominal value $Q \mathbf{E}$, that a particulate of charge $Q$ would experience in a field $\mathbf{E}$ in the absence of the plasma, may be called the "polarization force" since it is due solely to the sheath polarization (neglecting ion drag force). In particular, a spherically symmetric Debye sheath would not shield the external electric field at all (although it does shield the Coulomb field of the particulate that it surrounds).

Although these characteristics of Debye shielding may be explained (see Sec. VI below) intuitively, it is not a trivial matter to demonstrate them rigorously. Estimating the total force on a particulate involves not only calculation of the electrostatic sheath-polarization force, but also the contribution of pressure forces on the particulate due to the ambient plasma. It is not obvious that this pressure force and the sheath-polarization force are both small for a small particulate. The pressure force is due mainly to the positive ions, which are electrostatically attracted by the negatively charged particulate (in this sense, the pressure force also has electrostatic origins). Generally, the ion density increases toward the particulate, and for a small particulate in a uniform plasma the spherical symmetry of the particulate ensures zero net force due to the ion pressure. In a nonuniform plasma, however, a slight imbalance between the pressure force on one side of the particulate and that on the other may result in a finite net force even in the limit of zero particulate size. In other words, one needs detailed calculations of the pressure force to determine its magnitude, even for an infinitesimal particulate.

We now estimate the magnitude of the polarization force. As mentioned above, in a nonuniform plasma the 
Debye length $\lambda_{D}$ (which represents the thickness of the sheath) is a function of position, and the sheath around a particulate is no longer spherically symmetric. The deformed (i.e., polarized) sheath creates an electric field at the particulate location, and exerts a force-the polarization force-in addition to the external-field force $Q \mathbf{E}$.

If the Debye length is $\lambda_{D}=20 \mu \mathrm{m}$ and the scale length for macroscopic variations is $L=1 \mathrm{~cm}$ (typical values for materials-processing plasmas) the variation of macroscopic plasma variables, such as the electrostatic potential, over the sheath is very small, of order $\lambda_{D} / L=2 \times 10^{-3}$. However, even small charge separations may create large electric fields. Let us assume that the sheath is polarized due to the plasma nonuniformity, creating an excess charge $Q \lambda_{D} / L$ at one end of the sheath and $-Q \lambda_{D} / L$ at the other (the particulate has charge $-Q$, so the sheath contains the opposite charge $Q$ more or less within a sphere of radius $\lambda_{D}$ ). This charge separation creates an electric field, which exerts a force $F_{1}$ on the particulate, given roughly by

$$
F_{1} \simeq Q \frac{Q \lambda_{D} / L}{\varepsilon_{0} \lambda_{D}^{2}} .
$$

Comparing the polarization force $F_{1}$ with the direct electric-field force $F_{0}=Q E$, where we take $E \simeq k_{B} T_{e} / e L$ ( $e$ and $T_{e}$ being the electron charge and temperature), we obtain

$$
\frac{F_{1}}{F_{0}} \simeq \frac{e Q}{\varepsilon_{0} \lambda_{D} k_{B} T_{e}}=\frac{Z_{D}}{\lambda_{e}^{2} \lambda_{D} n_{e}} .
$$

Here $n_{e}$ is the electron density, $Q=Z_{D} e$, and we have used the definition of the electron Debye length $\lambda_{e}=\left(\varepsilon_{0} k_{B} T_{e} / e^{2} n_{e}\right)^{1 / 2}$. Introducing the plasma parameter $g=1 / \lambda_{e}^{3} n_{e}$, which is typically small $-g \ll 1$ is indeed a condition for a system of charged particles to be a "plasma" - we obtain

$$
\frac{F_{1}}{F_{0}} \simeq g Z_{D} \frac{\lambda_{e}}{\lambda_{D}} .
$$

Typically, $Z_{D} \gg 1$ and $\lambda_{e} \gtrsim \lambda_{D}$, so the ratio $F_{1} / F_{0}$ may be of order 1, i.e., the polarization force $F_{1}$ may be comparable to the direct electrostatic force $F_{0}=Q E$. To determine the magnitude of $F_{1}$ more accurately, one needs a detailed analysis, which will be presented below.

For the sake of simplicity, we do not consider effects of a finite plasma flow in this paper. The plasma flow, especially ion flow, influences the transport of particulates in two ways. One is to exert an ion drag force, which represents the effect of Coulomb collisions between ions and the charged grain, and incurs a change of the ion velocity distribution function. The other is a fluidlike effect, by which the ion flow modifies the shape of the Debye sheath. In particular, when the ion flow velocity is sufficiently large compared to the ion thermal velocity $v_{\text {th }}=\left(k_{B} T_{i} / m_{i}\right)^{1 / 2}$, where $T_{i}$ and $m_{i}$ are the ion temperature and mass, the ions are unable to form a sheath around the particulate and consequently the Debye sheath consists only of a deficiency of electrons (the ion sheath is "blown away" by the strong ion flow). Under such conditions, the ion density distribution is almost uniform in the sheath. This fluidlike effect does not involve a change of the ion velocity distribution function.

A comprehensive study of drag forces on a particulate in a uniform plasma has recently been given by Northrop and Birmingham [12], where large-angle Coulomb scattering [13], dynamical friction $[14,15]$, and collective effects [16] are treated systematically based on the theory of Hubbard [17] and Kihara and Aono [18]. The kinetic theory of Northrop and Birmingham simultaneously deals with both effects-drag force and fluidlike effects-mentioned above for a uniform plasma. For a nonuniform plasma, with which we are concerned here, a similar kinetic treatment is more complex and beyond the scope of the present work. However, a more detailed study of fluid-particulate interactions in a nonuniform plasma, deferred to a future publication, generalizes the results of this paper and shows that the sheath deformation due to a finite ion flow (i.e., the fluidlike effect mentioned above) has only a minor influence on the force exerted on particulates. In other words, the sum of the force presented in this paper and the usual ion drag force [13] accounts for the particulate transport in a nonuniform plasma. Effects of a finite flow will be discussed again in Sec. VIII.

This paper is organized as follows. In Sec. II we introduce our model of a charged particulate immersed in a nonuniform plasma. The electrostatic force is then obtained for infinitesimal and finite-radius particulates in Secs. III and IV. Noting that, in the absence of a finite plasma flow, the total force on the particulate is only the sum of the electrostatic force and pressure force, we derive the pressure force and total force in Sec. V. In Sec. VI, a physical interpretation for the results obtained in Secs. III-V is presented, and in Sec. VII the magnitude of the polarization force is estimated under realistic glow discharge conditions. Finally, Sec. VIII summarizes our results and briefly discusses effects of finite ion flows on the particulate force.

\section{FORMULATION}

We consider an unmagnetized plasma, in which the electrostatic potential, ion and electron densities, and ion flow velocity at position $\mathbf{r}$ are given by $\Psi_{0}(\mathbf{r}), n_{i 0}(\mathbf{r})$, $n_{e 0}(\mathbf{r})$, and $\mathbf{v}_{i 0}(\mathbf{r})$. Here the subscript 0 indicates macroscopic variables that vary weakly over the small scale of the ion or electron Debye lengths. The mean charge density is then $\rho_{0}=q n_{i 0}-e n_{0}$, where the ion and electron charges are denoted by $q$ and $-e$ (we assume ions of single species; the extension to multiple species is straightforward). The potential satisfies Poisson's equation $\Delta \Psi_{0}=-\rho_{0} / \varepsilon_{0}$. The plasma and electric field are sustained by some external means, such as a voltage applied to the electrodes. In this sense, the field may be called an externally applied field, although the plasma adjusts itself to determine the electric field profile, $\mathbf{E}_{0}(\mathbf{r})=-\nabla \Psi_{0}$.

If we place a small particulate at $\mathbf{r}=0$ in this plasma, it will acquire a negative charge $-Q$, due to the attachment of high-mobility plasma electrons. The charged particulate perturbs the local electric field and plasma 
density, forming a Debye sheath around it. The resultant field potential $\Psi(\mathbf{r})$ again satisfies Poisson's equation:

$$
\Delta \Psi(\mathbf{r})=-\frac{\rho(\mathbf{r})}{\varepsilon_{0}},
$$

where $\rho(r)$ denotes the total charge density given by

$$
\rho(\mathbf{r})=-Q \delta(\mathbf{r})+q n_{i}(\mathbf{r})-e n_{e}(\mathbf{r}),
$$

and $n_{i}(\mathbf{r})$ and $n_{e}(\mathbf{r})$ are ion and electron densities after the perturbation. Here the particulate is assumed to be infinitesimally small. The boundary conditions are given by

$$
\Psi(\mathbf{r}) \rightarrow \Psi_{0}(\mathbf{r}) \text { as }|\mathbf{r}| \rightarrow \infty .
$$

The dependence of the ion and electron densities on the electrostatic field is determined by the microscopic plasma characteristics. If collisions are sufficiently frequent, it may be assumed that the plasma is in thermal equilibrium locally (i.e., on the scale of Debye lengths), and the local densities are then given by the Boltzmann distributions:

$$
\begin{aligned}
& n_{i}(\mathbf{r})=n_{i 0}(\mathbf{r}) \exp \left[\frac{-q \varphi(\mathbf{r})}{k_{B} T_{i}}\right], \\
& n_{e}(\mathbf{r})=n_{e 0}(\mathbf{r}) \exp \left[\frac{e \varphi(\mathbf{r})}{k_{B} T_{e}}\right],
\end{aligned}
$$

where

$$
\varphi(\mathbf{r})=\Psi(\mathbf{r})-\Psi_{0}(\mathbf{r})
$$

is the perturbation in the potential, and $T_{i}$ and $T_{e}$ are the ion and electron temperatures. The macroscopic variables $n_{i 0}(\mathbf{r}), n_{e 0}(\mathbf{r})$, and $\Psi_{0}(\mathbf{r})$ are slowly varying functions of $r$, in the sense that $\left|\nabla \Psi_{0}(\mathbf{r})\right| \ll|\nabla \varphi(r)|$, etc.

If the potential perturbation is sufficiently small (i.e., $\left.|\varphi|<<k_{B} T_{i}, k_{B} T_{e}\right)$, the ion and electron densities given in Eqs. (4) and (5) may be linearized with respect to $\varphi$ (the so-called Debye-Hückel approximation). Substituting the resulting expressions for $n_{i}(\mathbf{r})$ and $n_{e}(\mathbf{r})$ into Eq. (1) and subtracting Poisson's equation for the mean field, $\Delta \Psi_{0}=-\rho_{0} / \varepsilon_{0}$, we obtain

$$
\Delta \varphi(\mathbf{r})=\frac{Q}{\varepsilon_{0}} \delta(\mathbf{r})+k_{D}^{2}(\mathbf{r}) \varphi(\mathbf{r}),
$$

with

$$
k_{D}^{2}(\mathbf{r})=\frac{q^{2} n_{i 0}(\mathbf{r})}{k_{B} T_{i}}+\frac{e^{2} n_{e 0}(\mathbf{r})}{k_{B} T_{e}} .
$$

The boundary condition for Eq. (6) is then given by

$$
\varphi(\mathbf{r}) \rightarrow 0 \text { as }|\mathbf{r}| \rightarrow \infty \text {. }
$$

The characteristic Debye length $\lambda_{D}(\mathbf{r})$, defined by $\lambda_{D}(\mathbf{r})=k_{D}^{-1}(\mathbf{r})$, is in general spatially dependent. In terms of the ion and electron Debye lengths

$$
\lambda_{i}(\mathbf{r})=\left[\frac{k_{B} T_{i}}{q^{2} n_{i 0}(\mathbf{r})}\right]^{1 / 2}
$$

and

$$
\lambda_{e}(\mathbf{r})=\left[\frac{k_{B} T_{e}}{e^{2} n_{e 0}(\mathbf{r})}\right]^{1 / 2},
$$

we have the relation

$$
\lambda_{D}(\mathbf{r})=\left[\frac{1}{\lambda_{i}^{2}(\mathbf{r})}+\frac{1}{\lambda_{e}^{2}(\mathbf{r})}\right]^{-1 / 2} .
$$

Note, in particular, that $\lambda_{D} \simeq \lambda_{i}$ if $T_{e} \gg T_{i}$.

The assumption of the Boltzmann distribution for ions given in Eq. (4), and its linearization employed to derive Eq. (6) -i.e., the Debye-Hückel approximation-need further explanation. In typical laboratory or space plasmas, where the ion Debye length is significantly smaller than the ion mean free path, the ion Boltzmann distribution inside the sheath of a particulate is rather unrealistic. Also, for a particulate in a relatively cold plasma (e.g., $T_{i}<1 \mathrm{eV}$ ), the electrostatic potential at the surface of the particulate is larger than the ion kinetic energy, i.e., $\varphi(a) \gtrsim k_{B} T_{i}$, which violates the condition for linearization. Therefore, rigorously speaking, the model we have adopted does not apply to systems of dust grains in laboratory plasmas.

However, a simple physical argument suggests that, despite the apparent invalidity of the model, the properties of the system that we are concerned with in this system are relatively insensitive to the exact form of the ion distribution in the sheath. This insensitivity explains why the Debye-Hückel approximation has been successfully applied to various systems, ranging from colloidal particles [19] to dust in space plasmas [20,21], in earlier studies.

The important physical aspect of a particulate sheath is that, toward the particulate surface, the ion density increases monotonically whereas the electron density decreases monotonically (for collisionless plasmas, see [22]). This behavior is retained by the linearized ion distribution $n_{i}(\mathbf{r})=n_{i 0}\left[1-q \varphi(\mathbf{r}) / k_{B} T_{i}\right]$, noting that the asymptotic behavior of the potential, $\varphi \rightarrow-\infty$ monotonically as $|\mathbf{r}| \rightarrow 0$, is self-consistently derived from the linearized Boltzmann distributions. The thickness of the ion-excess layer (i.e., ion sheath) and electron-deficient layer (i.e., electron sheath) - which overlap each other - are typically given by the ion and electron Debye lengths. The exact value of the ion-sheath thickness, of course, depends on a specific model of the ion density distributions. See Appendix A for further discussion of this point.

Furthermore, when the mean (i.e., flow) ion velocity exceeds the thermal velocity $v_{\text {th }}=\left(k_{B} T_{i} / m_{i}\right)^{1 / 2}$, as is often the case in laboratory plasmas, the ion sheath may either be strongly distorted or unable to form at all around the particulate. In this case, the sheath is made up only of a deficiency of electrons and its thickness is given approximately by the electron Debye length $\lambda_{e}$. Note that, in typical process plasmas, the plasma flow velocity may reach the ion sound velocity $c_{s}=\left(k_{B} T_{e} / m_{i}\right)^{1 / 2}$ but seldom exceeds the electron thermal velocity $v_{e, \text { th }}=\left(k_{B} T_{e} / m_{e}\right)^{1 / 2}$. Under such conditions, the ion density distribution is given neither by the 
Boltzmann distribution Eq. (4) nor the collisionless distribution derived by Bernstein and Rabinowitz [22]: it is almost uniform in the sheath. Again, the exact form of the ion density distribution does not influence the qualitative form of the potential profile and the plasma pressure near the particulate. We shall discuss the effects of a finite ion flow further in Sec. VIII.

Since the final results are likely insensitive to the exact functional form assumed for the ion density distribution, the advantage of using the linearized Boltzmann form is obvious: the system is solvable analytically and thus yields clear insight into its physical behavior. The zeroth-order solution that we employ in the following is the spherically symmetric solution to the Debye-Hückel approximation of Poisson's equation, which has been studied extensively and is well understood. Of course, studying the system numerically with more realistic ion density distribution is important [23,24], and more simulation studies are needed. Our analytical model serves as a reference system and point of departure for the subsequent construction of models under more realistic conditions.

\section{ELECTROSTATIC POTENTIAL}

We now solve Poisson's equation (6) to obtain the electric-field potential around the particulate. Here the spatial dependence of $k_{D}=\lambda_{D}^{-1}$ is assumed to be weak, in the sense that $\left|\nabla k_{D}\right| k_{D} \sim \delta \equiv \lambda_{D} / L$, with $L$ being the macroscopic scale length. Therefore, we may expand the function $k_{D}^{2}(\mathbf{r})$ around the particulate position $\mathbf{r}=0$ in a Taylor series to obtain

$$
k_{D}^{2}(\mathbf{r})=k_{0}^{2}+\mathbf{r} \cdot \nabla k_{D}^{2},
$$

where $k_{0}=\lambda_{0}^{-1}=k_{D}(0)$ is the inverse Debye length at $\mathbf{r}=0$, and $\nabla k_{D}^{2}$ is also evaluated at $\mathbf{r}=0$. Taking the direction of $\nabla k_{D}^{2}$ as the $z$ direction, we may write

$$
k_{D}^{2}(z)=k_{0}^{2}+\sigma z,
$$

where $\sigma=d k_{D}^{2} / d z$. In terms of the Debye length $\lambda_{D}$, we have

$$
\nabla k_{D}^{2}=-\frac{2}{\lambda_{0}^{3}} \nabla \lambda_{D},
$$

or $\sigma=-2\left(d \lambda_{D} / d z\right) / \lambda_{D}^{3}$, evaluated at $\mathbf{r}=0$.

Since the second term of Eq. (8) is smaller than the first (i.e., $\sigma z / k_{0}^{2} \sim \delta$ ), we may solve Eq. (6) using a perturbation method: we write $\varphi(\mathbf{r})=\varphi^{(0)}(\mathbf{r})+\varphi^{(1)}(\mathbf{r})$, where the zeroth-order solution $\varphi^{(0)}$ satisfies

$$
\Delta \varphi^{(0)}(\mathbf{r})=\frac{Q}{\varepsilon_{0}} \delta(\mathbf{r})+k_{0}^{2} \varphi^{(0)}(\mathbf{r}),
$$

and the first-order correction $\varphi^{(1)}(\mathbf{r})$ satisfies

$$
\Delta \varphi^{(1)}(\mathbf{r})-k_{0}^{2} \varphi^{(1)}(\mathbf{r})=\sigma z \varphi^{(0)}(\mathbf{r})
$$

The appropriate boundary conditions are $\varphi^{(0)}, \varphi^{(1)} \rightarrow 0$ as $|\mathbf{r}| \rightarrow \infty$.

The solution to the zeroth-order equation (10) is the well-known screened Coulomb potential

$$
\varphi^{(0)}(\mathbf{r})=-\frac{Q}{4 \pi \varepsilon_{0}} \frac{\exp \left(-k_{0} r\right)}{r},
$$

where $r=|\mathbf{r}|$. With the use of this expression for $\varphi^{(0)}$, the solution to the first-order equation (11) is given by

$$
\varphi^{(1)}(\mathbf{r})=\frac{\sigma Q}{16 \pi \varepsilon_{0} k_{0}} z \exp \left(-k_{0} r\right)
$$

One can easily confirm Eq. (13) by substitution into Eq. (11). For a more systematic derivation of $\varphi^{(1)}$, see Appendix B.

The total charge density $\rho(\mathbf{r})$ is then given by

$$
\rho(\mathbf{r})=\rho_{0}(\mathbf{r})-Q \delta(\mathbf{r})+\rho_{s}(\mathbf{r}),
$$

where $\rho_{s}(\mathbf{r})$ is the charge density of the Debye sheath, given by

$$
\begin{aligned}
\rho_{s}(\mathbf{r})= & -\varepsilon_{0} k_{0}^{2}\left[\varphi_{0}(\mathbf{r})+\varphi_{1}(\mathbf{r})\right]-\varepsilon_{0} \sigma z \varphi_{0}(\mathbf{r}) \\
=\frac{k_{0}^{2} Q}{4 \pi}\left[\frac{\exp \left(-k_{0} r\right)}{r}\right. & \\
& \left.+\left(\frac{1}{r}-\frac{k_{0}}{4}\right) \frac{\sigma \exp \left(-k_{0} r\right)}{k_{0}^{2}} z\right] .
\end{aligned}
$$

In Eq. (14) we have retained terms to first order in $\delta=\lambda_{D} / L$.

Figure 1 shows the contour lines of the potential $\varphi=\varphi^{(0)}+\varphi^{(1)}$ given by Eqs. (12) and (13). The closed circle at the center represents the position of the particulate. Here the potential is normalized by $Q k_{0} / 4 \pi \varepsilon_{0}$, and the parameter $\sigma / k_{0}^{3}\left(\simeq \lambda_{D} / L\right)$ is chosen to be 0.01 . Note that the contours are at logarithmic intervals. As is clearly seen, the Debye length $\lambda_{D}(z)$ is smaller for larger $z$, resulting in a positive potential-overshooting in $\varphi$ for $z / \lambda_{D} \gtrsim 20$. Similarly, Fig. 2 shows the charge-density distribution around the particulate under the same condi-

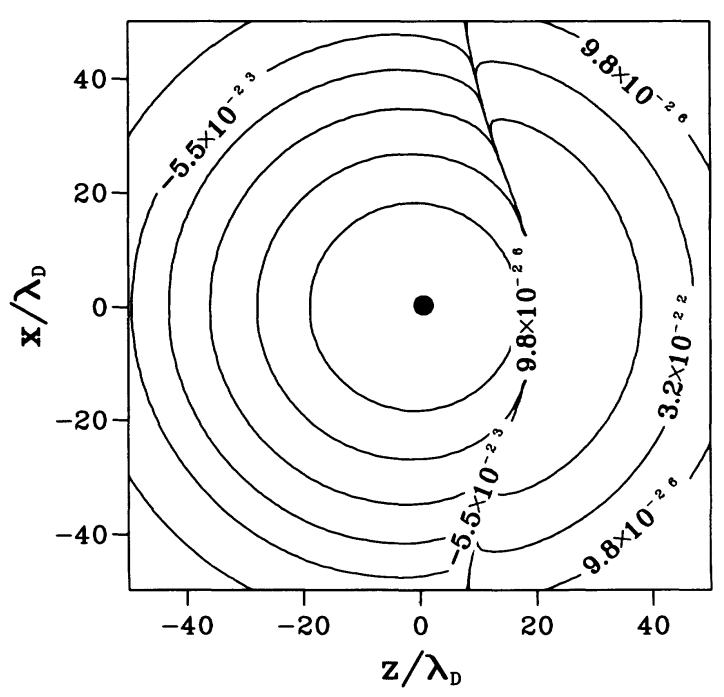

FIG. 1. Contour lines (on a logarithmic scale) of the sheath potential $\varphi=\varphi^{(0)}+\varphi^{(1)}$ given by Eqs. (12) and (13). $\nabla n$ is in the $z$ direction $(\sigma>0)$. The mean-field potential $\Psi_{0}$ is not depicted. The potential is normalized by $Q k_{0} / 4 \pi \varepsilon_{0}$. 


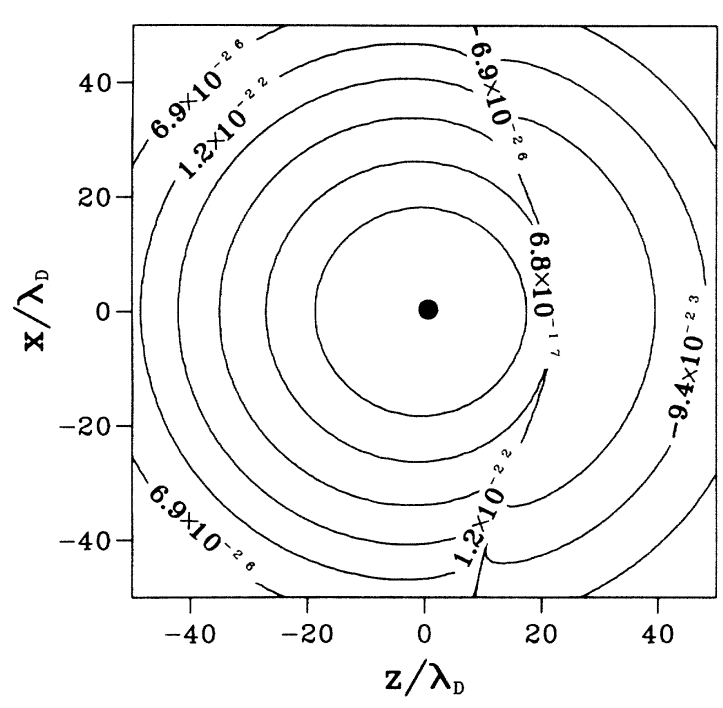

FIG. 2. Contour lines (on a logarithmic scale) of the sheath charge density $\rho_{s}$ given by Eq. (14) under the same conditions as those in Fig. 1. The background charge density $\rho_{0}$ is not depicted. The charge density is normalized by $Q k_{0}^{3} / 4 \pi$.

tions. The charge density is seen to be negative for $z / \lambda_{D} \gtrsim 20$, indicating polarization of the Debye sheath.

The dipole moment of the particulate-sheath system is given by

$$
\mathbf{P}=\int \mathbf{r} \rho_{s}(\mathbf{r}) d \mathbf{r},
$$

which yields $\mathbf{P}=0$ upon substitution of $\rho_{s}$ as in Eq. (14). In other words, the particulate-sheath system possesses no dipole moment, as one might expected (note that the plasma sheath can "shield" any multipole moment, including a monopole, i.e., a bare charge).

The electrostatic force on the particulate may be calculated as

$$
\mathbf{F}^{\mathrm{ES}}=Q \boldsymbol{\nabla}\left[\Psi(\mathbf{r})-\frac{-Q}{4 \pi \varepsilon_{0} r}\right],
$$

where $\Psi=\Psi_{0}+\varphi^{(0)}+\varphi^{(1)}$, and the second term in the parentheses represents the self-field due to the particulate charge $-Q$ that needs to be subtracted for the force calculation. Since this self-field and $\varphi^{(0)}$ are spherically symmetric, the only potentials that contribute to the net force are $\Psi_{0}$ and $\varphi^{(1)}$. Writing $\mathbf{E}_{0}=-\nabla \Psi_{0}$ and using Eq. (13), we obtain

$$
F_{z}^{\mathrm{ES}}=-Q E_{0}+\frac{\sigma Q^{2}}{16 \pi \varepsilon_{0} k_{0}} .
$$

Here $F_{z}^{\mathrm{ES}}$ and $E_{0 z}$ denote the $z$ components of $\mathrm{F}^{\mathrm{ES}}$ and $\mathbf{E}_{0}$.

As will be shown in Sec. $V$, the pressure force $F^{p}$ on the infinitesimal particulate vanishes, so Eq. (15) indeed gives the total force in the absence of mean ion flow (i.e., there is no drag force). Using Eq. (9) the total force, to $O(\delta)$, may be written in vector form as

$$
\mathbf{F}=-Q \mathbf{E}_{0}-\frac{Q^{2} \nabla \lambda_{D}}{8 \pi \varepsilon_{0} \lambda_{D}^{2}} .
$$

The (Helmholtz) free energy $F_{H}$, which may be calculated from $F_{H}=-\int^{z} F_{z} d z$, is then given by

$$
F_{H}=-Q \Psi_{0}-\frac{Q^{2}}{8 \pi \varepsilon_{0} \lambda_{D}},
$$

where $\Psi_{0}$ and $\lambda_{D}$ are evaluated at the particle position. In Eq. (17), the free energy of the unperturbed system is set to zero. Note that the polarization force-the second term of Eq. (16) - is always in the direction of decreasing Debye length, regardless of the sign of the particulate charge. In other words, as may be seen from Eq. (17), the particulate has a lower free energy when it has a thinner Debye sheath (i.e., smaller $\lambda_{D}$ ).

\section{FINITE-RADIUS EFFECTS}

We now extend the preceding calculation to particulates of finite radius. For simplicity, we assume that the particulate is a perfect spherical conductor of radius $a$. The radius $a$ may be comparable to the Debye length $\lambda_{D}$ but should be significantly smaller than the macroscopic scale length (i.e., $a, \lambda_{D} \ll L$ ). The assumptions regarding plasma properties made in Sec. II are also employed in this section.

The problem may be formulated in the same manner as in Sec. II, except that the total charge is now given by

$$
\rho(\mathbf{r})=q n_{i}(\mathbf{r})-e n_{e}(\mathbf{r})
$$

in the plasma, and the boundary conditions are

$$
\begin{aligned}
& \Psi(\mathbf{r}) \rightarrow \Psi_{0}(\mathbf{r}) \text { as } r \rightarrow \infty, \\
& \Psi(\mathbf{r})=\Psi_{0}(\mathbf{0})+V_{s} \text { at } r=a
\end{aligned}
$$

instead of Eqs. (2) and (3). In Eq. (19), $V_{s}$ denotes a constant voltage on the particulate surface (measured from the background potential $\Psi_{0}$ at $\mathbf{r}=0$ ), which is a function of the total charge $-Q$ residing on the surface through the relation

$$
Q=\varepsilon_{0} \oint_{r=a} \frac{\partial \Psi}{\partial r} d S,
$$

the integration being over the particulate surface (note that the quantity $-\varepsilon_{0} \partial \Psi /\left.\partial r\right|_{r=a}$ represents the surface charge density).

Writing $\varphi(\mathbf{r})=\varphi^{(0)}(\mathbf{r})+\varphi^{(1)}(\mathbf{r})$ again, the zeroth-order solution now satisfies

$$
\Delta \varphi^{(0)}(\mathbf{r})=k_{0}^{2} \varphi^{(0)}(\mathbf{r}),
$$

with the boundary conditions $\chi^{(0)} \rightarrow 0$ as $r \rightarrow \infty$ and $\varphi^{(0)}=V_{s}$ at $r=a$. Here we have assumed that $\Psi_{0}(z)=\Psi_{0}(0)-E_{0} z$, neglecting $O\left(\delta^{2}\right)$ terms $\left(\delta=\lambda_{D} / L\right.$ as before). The solution to Eq. (21) is readily obtained as

$$
\varphi^{(0)}(\mathbf{r})=\frac{V_{s} a \exp \left[-k_{0}(r-a)\right]}{r} .
$$

The first-order equation for $\varphi^{(1)}$ is given by Eq. (11), 
but with boundary conditions $\varphi^{(1)} \rightarrow 0$ as $r \rightarrow \infty$ and $-E_{0} a \cos \theta+\varphi^{(1)}(\mathbf{r})=0$ at $r=a$. Here $\theta$ denotes the polar angle measured from the $z$ axis. Substituting Eq. (22) into Eq. (11) and solving the resulting equation, we obtain

$$
\begin{aligned}
\varphi^{(1)}(\mathbf{r})= & E_{0}\left[\frac{a}{r}\right)^{3}\left(\frac{1+k_{0} r}{1+k_{0} a}\right) \exp \left[-k_{0}(r-a)\right] z \\
- & \frac{\sigma a V_{s}}{4 k_{0}}\left[\frac{a}{r}\right)^{3}\left(\frac{1+k_{0} r}{1+k_{0} a}-\frac{r^{3}}{a^{3}}\right) \\
& \times \exp \left[-k_{0}(r-a)\right] z,
\end{aligned}
$$

where $z=r \cos \theta$. It is straightforward to confirm that the above expression for $\varphi^{(1)}$ indeed satisfies Eq. (11). Note that $\varphi^{(1)}=E_{0} a \cos \theta$ on the surface of the particulate, which also satisfies the boundary condition stated above. A systematic derivation of Eq. (23) from Eq. (11) is given in Appendix B.

To relate the potential $V_{s}$ to the particle charge $-Q$, we now substitute $\Psi=\Psi_{0}+\varphi^{(0)}+\varphi^{(1)}$ into Eq. (20) to obtain $V_{s}=-Q / 4 \pi \varepsilon_{0}\left(1+k_{0} a\right) a$, or

$$
\varphi^{(0)}(\mathbf{r})=-\frac{Q \exp \left[-k_{0}(r-a)\right]}{4 \pi \varepsilon_{0}\left(1+k_{0} a\right) r}
$$

and

$$
\begin{aligned}
\varphi^{(1)}(\mathbf{r})= & E_{0}\left[\frac{a}{r}\right]^{3}\left(\frac{1+k_{0} r}{1+k_{0} a}\right) \exp \left[-k_{0}(r-a)\right] z \\
& \left.\left.+\frac{\sigma Q}{16 \pi \varepsilon_{0}\left(1+k_{0} a\right) k_{0}}\right) \frac{a}{r}\right]^{3} \\
& \times\left(\frac{1+k_{0} r}{1+k_{0} a}-\frac{r^{3}}{a^{3}}\right) \exp \left[-k_{0}(r-a)\right] z .
\end{aligned}
$$

The electrostatic force exerted on the particulate is given by

$$
\begin{aligned}
\mathbf{F}^{\mathrm{ES}} & =\int_{r \leq a} \rho(\mathbf{r}) \mathbf{E}(\mathbf{r}) d \mathbf{r} \\
& =\varepsilon_{0} \oint_{r=a}\left[\mathbf{E}(\mathbf{E} \cdot \hat{\mathbf{n}})-\frac{1}{2} \mathbf{E}^{2} \hat{\mathbf{n}}\right] d S,
\end{aligned}
$$

where $\mathbf{E}=-\nabla \Psi, \hat{\mathbf{n}}$ is an outward unit normal vector on the particulate surface, and the above integral is taken over the entire surface. Substituting $\mathbf{E}=\mathbf{E}_{0}$ $-\nabla\left(\varphi^{(0)}+\varphi^{(1)}\right)$, we obtain

$$
F_{z}^{\mathrm{ES}}=Q E_{0}\left[1+\frac{k_{0}^{2} a^{2}}{3\left(1+k_{0} a\right)}\right]+\frac{\sigma Q^{2}\left(1+2 k_{0} a / 3\right)}{16 \pi \varepsilon_{0}\left(1+k_{0} a\right)^{2} k_{0}} .
$$

Note that Eq. (26) agrees with Eq. (15) when $k_{0} a=0$.

In the case of a finite-radius particulate, the external electric field $\mathbf{E}_{0}$ determines the charge distribution on the particulate's conducting surface, i.e., the polarization of the particulate. The polarized surface charges then create an electric field represented by the first term in Eq. (25), which in turn exerts an electrostatic force on the particulate given by the second term in the bracket of Eq. (26). Note that this force, which exists even in the case $\sigma=0$, is unrelated to the polarization of the sheath. This particulate-polarization field, however, also exerts a force on the plasma, increasing the ion pressure on the particulate. As will be shown in Sec. V the ion pressure force exactly cancels this particulate-polarization force [but not the sheath-polarization force given by the second term of Eq. (25)].

\section{PRESSURE FORCES}

Besides the drag force, the only forces that are exerted on a particulate are the direct electrostatic force (which was derived in previous sections) and the pressure force due to the ambient plasma. As discussed in Sec. I, the magnitude of the pressure force is not obviously negligible, especially in the case of a finite-radius particulate. The pressure force is also strongly influenced by the electrostatic field around the particulate, since the densities of ions and electrons are determined by the local electric field. Thus, in order to assess the effects of an external electric field, the pressure force must be taken into account self-consistently.

Expanding Eq. (4), the ion pressure may be written as

$$
\begin{aligned}
p_{i}(\mathbf{r})=k_{B} T_{i} n_{i}(\mathbf{r})= & k_{B} T_{i} n_{i 0}(\mathbf{r})-q n_{i 0}(\mathbf{r}) \varphi \\
& +\frac{1}{2} q^{2} \beta_{i} n_{i 0}(\mathbf{r}) \varphi^{2},
\end{aligned}
$$

where $\beta_{i}=1 /\left(k_{B} T_{i}\right)$. A similar expression holds for the electron pressure, using Eq. (5). The sum of these pressure expressions yields the total pressure

$$
p(\mathbf{r})=p_{0}(\mathbf{r})-\rho_{0}(\mathbf{r}) \varphi+\frac{\varepsilon_{0}}{2} k_{D}^{2}(\mathbf{r}) \varphi^{2} .
$$

Here we have taken the expansion to order $\varepsilon^{2}$, where $\varepsilon \sim e \beta_{e} \varphi$, since the third term in Eq. (27) may be dominant near the particulate surface. To see this more clearly, we cast Eq. (27) in dimensionless form as

$$
\widetilde{p}(\mathbf{r})=\widetilde{p}_{0}(\mathbf{r})-\frac{\rho_{0}(\mathbf{r})}{q n_{e 0}(0)} \widetilde{\varphi}+\frac{1}{2} k_{D}^{2}(\mathbf{r}) \lambda_{e}^{2} \widetilde{\varphi}^{2}
$$

where

$$
\widetilde{\varphi}=e \beta_{e} \varphi \text { and } \widetilde{p}=\beta_{e} p / n_{e 0}(0) .
$$

In Eq. (28), note that $k_{D}^{2}(\mathbf{r}) \lambda_{e}^{2}=O(1)$, while the coefficient of the second term is $\rho_{0} / q n_{e 0}(0)=O\left(\delta^{2}\right)$, where $\delta=\lambda_{e} / L$, since $\rho_{0} \simeq \varepsilon_{0} \Psi_{0} / L^{2}$ and $\Psi_{0} \simeq 1 / e \beta_{e}$. Thus, since $\widetilde{\varphi}=O(\varepsilon)$, the third term dominates over the second term if $\delta=\lambda_{e} / L \sim \varepsilon$. In particular, in the limit of a uniform plasma, the first and second terms in Eq. (28) vanish, and the pressure is due solely to the third term, i.e., to ions and electrons in the sheath.

Therefore, assuming that $\delta=\lambda_{e} / L \sim \varepsilon$ and taking terms to $O\left(\varepsilon^{3}\right)$ in Eq. (28), we may rewrite Eq. (27) as

$$
p(\mathbf{r})=p_{0}(z)-\rho_{0}(0) \varphi+\frac{\varepsilon_{0}}{2}\left(k_{0}^{2}+\sigma z\right) \varphi^{2},
$$

where for the background pressure $p_{0}(z)$ we take its Taylor expansion around $\mathbf{r}=0$ only up to $O\left(\varepsilon^{3}\right)$. In Eq. (29), the background quantities are assumed to depend only on 
$z$, as before.

The pressure force on the particulate is then calculated from

$$
\mathbf{F}^{p}=-\oint_{p \hat{\mathbf{n}}} d S
$$

where the integration is taken over the particulate surface with outward normal unit vector $\hat{\mathbf{n}}$. The $z$ component of $\mathbf{F}^{p}$ may be expressed as

$$
F_{z}^{p}=-2 \pi a^{2} \int_{0}^{\pi} p \cos \theta \sin \theta d \theta
$$

Substituting Eq. (29) into Eq. (30), we obtain

$$
\begin{aligned}
F_{z}^{p}= & -\frac{4}{3} \pi a^{3} p_{0}^{\prime}-\frac{2}{15} \pi a^{2} p_{0}^{\prime \prime \prime} \\
& +\frac{4}{3} \pi a^{3} \rho_{0} E_{0}-\frac{Q E_{0}\left(k_{0} a\right)^{2}}{3\left(1+k_{0} a\right)} \\
& -\frac{\sigma a Q^{2}}{24 \pi \varepsilon_{0}\left(1+k_{0} a\right)^{2}}-\frac{2}{5} \pi a^{2} \varepsilon_{0} \sigma E_{0}^{2}
\end{aligned}
$$

In deriving Eq. (31) we use $\varphi^{(0)}=Q / 4 \pi \varepsilon_{0}\left(1+k_{0} a\right) a$ and $\varphi^{(1)}=E_{0} a \cos \theta$. Note that the magnitudes of $\varphi^{(0)}$ and $\varphi^{(1)}$ are of the same order at the surface, i.e., $e \beta_{e} \varphi^{(0)} \sim e \beta_{e} \varphi^{(1)} \sim \varepsilon$, since $E_{0} \sim 1 / e \beta_{e} L$ and $a \lesssim \lambda_{e}$.

If the particulate radius is sufficiently small compared to the Debye length (i.e., $a \ll<\lambda_{e}$ ), which is usually the case for laboratory or space plasmas, it suffices to take terms up to $O\left(k_{0}^{2} a^{2}\right)$ in Eq. (31):

$$
F_{z}^{p}=-\frac{Q E_{0}\left(k_{0} a\right)^{2}}{3\left(1+k_{0} a\right)}-\frac{\sigma a Q^{2}}{24 \pi \varepsilon_{0}\left(1+k_{0} a\right)^{2}} .
$$

The first term above represents the pressure force due to the sheath-polarization field. As mentioned in Sec. III, this pressure force vanishes as $a \rightarrow 0$.

Adding Eqs. (26) and (32), we have the total force on a small particulate (i.e., $a<<\lambda_{e}$ ): $F_{z}=F_{z}^{\mathrm{ES}}+F_{z}^{p}$. In vector form, this may be written as

$$
\mathbf{F}=-Q \mathrm{E}_{0}-\frac{Q^{2}}{8 \pi \varepsilon_{0}\left(1+k_{D} a\right)^{2}} \frac{\nabla \lambda_{D}}{\lambda_{D}^{2}},
$$

where all the plasma quantities (such as $\mathbf{E}_{0}$ and $\lambda_{D}$ ) are evaluated at the particulate position, i.e., $\mathbf{r}=\mathbf{0}$.

The free energy $F_{H}$ may be calculated from Eq. (33) in a manner similar to that used to obtain Eq. (17):

$$
\begin{aligned}
F_{H} & =-Q \Psi_{0}+\frac{Q^{2}}{8 \pi \varepsilon_{0} a\left(1+k_{D} a\right)} \\
& =-Q \Psi_{0}-\frac{Q^{2} k_{D}}{8 \pi \varepsilon_{0}\left(1+k_{D} a\right)}+\frac{Q^{2}}{8 \pi \varepsilon_{0} a},
\end{aligned}
$$

where $\Psi_{0}$ and $k_{D}=1 / \lambda_{D}$ are evaluated at the particulate position. The third term in Eq. (34) represents the selfenergy of the particulate, which becomes infinity as $a \rightarrow 0$. This self-energy is not included in Eq. (17); the first and second terms of Eq. (34) agree with Eq. (17) in the limit $a \rightarrow 0$.

\section{WHY DEBYE SHEATHS DO NOT "SHIELD" CHARGED PARTICLES FROM EXTERNAL FIELDS}

We have shown that, in the absence of plasma flows, the total force on a particulate is the sum of the direct electrostatic force exerted by the external field and the sheath-polarization force. In particular, in the case of uniform density $(\sigma=0)$, the total force on the charge $-Q$ is exactly $-Q \mathbf{E}_{0}$. Thus, although an unpolarized spherically symmetric sheath screens the Coulomb field of the charged particulate that it surrounds (Debye shielding), it does not screen the particulate from an externally applied field.

The notion of a "dressed particle" is often used to explain phenomena concerning charged particles in plasmas (e.g., [16]). This concept suggests that a charged particle and its oppositely charged Debye sheath be regarded as a single "system," and serves as a convenient reminder that the electric field due to the particle is only of short range. However, one should be careful not to infer that the sheath also serves to shield the particulate from external fields. As shown in Sec. III, the total dipole moment $\mathbf{P}$ of a "dressed particle," i.e., the system of a particulate and its sheath, is zero even in the presence of sheath polarization. One may thus "formally" calculate the force on this dipole due to an external field as $\mathbf{F}=\boldsymbol{\nabla} \mathbf{P} \cdot \mathbf{E}_{0}$, which is of course zero since $\mathbf{P}=\mathbf{0}$. Thus, this dipole force does not amount to the (total) force on the particle that we have derived above-contrary to what is postulated in [3].

One may also calculate the monopole moment-i.e., total charge - of a dressed particle, which is obviously zero since the total sheath charge is equal in magnitude but opposite in sign to that on the particle, regardless of whether or not the sheath is polarized. Thus, again, the "formal" force on the dressed particle is zero, which may be interpreted as perfect shielding of an external field by the sheath-i.e., the charged particle in a plasma does not move even in the presence of an external field, which again contradicts our earlier conclusions.

The source of this apparent contradiction is the misleading idea, suggested by the notion of a "dressed" particle, that the Debye sheath is somehow "permanently attached" to the particle (since the particle and sheath always move together). In fact, the Debye sheath is not attached to the particle. Even under the influence of an external electric field, ions and electrons in the sheath are in equilibrium under a balance between electrostatic forces (due both to external and sheath fields) and plasma pressure forces (due to thermal motion). If the external field is increased, the plasma pressure also increases to compensate for it, maintaining a net charge (i.e., a sheath) around the particle. (Hence, it is important to take pressure forces into account, especially in the case of finite-radius particles, as was done in Sec. IV.)

This should not be taken to imply, however, that the sheath is "attached" to the particle through pressure forces, so that the electrostatic force on the sheath is somehow transmitted to the particle. The sheath represents only a local perturbation of the background plasma: if the particle moves from $A$ to $B$, the plasma will relax to the unperturbed state about $A$ and a new 
perturbation centered at $B$ will arise. No net transport of matter is associated with such "movement" of the sheath. In other words, a dressed particle "undresses" and "dresses" each time it moves. Thus the presence of the sheath has no effect on the particle dynamics if the sheath is spherically symmetric: the only additional force arises from a polarization of the sheath. (If the particle velocity exceeds the ion thermal velocity, however, ions cannot "reform" a sheath sufficiently rapidly around the instant position of the particle, resulting in an ion "wake." In this paper we consider only the case of negligible plasma flows relative to the particle motion.)

\section{POLARIZATION FORCES IN A GI OW DISCHARGE}

As an application of the theory developed in Secs. II-V, we shall estimate the magnitude of the polarization force given by the second term of Eq. (16) under conditions representative of glow discharges used in plasma processing applications. In such discharges, the region adjacent to the electrode sheath (i.e., the "dark space") is called the presheath, in which a weak electric field accelerates ions and electrons while maintaining local charge neutrality.

A steady-state fluid description of the presheath region may be given as

$$
\begin{aligned}
& \frac{d}{d z}\left[n v_{i}(z)\right]=v_{\mathrm{ion}} n, \\
& \frac{d}{d z}\left[n v_{e}(z)\right]=v_{\mathrm{ion}} n, \\
& m_{i} v_{i} \frac{d v_{i}}{d z}=e E-m_{i} v_{\mathrm{ion}} v_{i}, \\
& 0=-k_{B} T_{e} \frac{d n}{d z}-e n E,
\end{aligned}
$$

where $v_{\text {ion }}$ is the electron-impact ionization rate. Equations (35) and (36) are continuity equations for ions and electrons, while Eqs. (37) and (38) are the corresponding momentum equations. We assume that the ion and electron flow velocities $\mathbf{v}_{i}$ and $\mathbf{v}_{e}$ and the electric field $\mathbf{E}$ are in the $z$ direction (with magnitudes $v_{i}, v_{e}$, and $E$, respectively), that the electron temperature $T_{e}$ is constant, and that charge neutrality holds approximately: $n=n_{i} \simeq n_{e}$. We also take ion charge $q=e$ and temperature $T_{i} \simeq 0$, and we set $m_{e} \simeq 0$ and assume that the ion-neutral-atom and electron-neutral-atom collision frequencies are negligibly small.

The small difference between ion and electron densities, $\delta n=n_{i}-n_{e}$, may be calculated from Poisson's equation

$$
\varepsilon_{0} \frac{d E}{d z}=e \delta n .
$$

All variables in Eqs. (35)_(38) are macroscopic quantities and are assumed to depend only on $z$. The subscript 0 that was used to indicate background plasma quantities in previous sections is omitted for simplicity.
From Eqs. (35) and (36), we obtain $v_{i}=v_{e}=v$. Writing $E=-d \Psi / d z$, and using normalized variables

$$
\phi=e \beta_{e} \Psi, \quad u=v / c_{s}, \quad \zeta=v_{\text {ion }} z / c_{s},
$$

$c_{s}=\left(k_{B} T_{e} / m_{i}\right)^{1 / 2}$ being the ion sound velocity, we rewrite Eqs. (35) $-(38)$ as

$$
\begin{aligned}
& \frac{d}{d \zeta}(n u)=n, \\
& \frac{d n}{d \zeta}=n \frac{d \phi}{d \zeta}, \\
& u \frac{d u}{d \zeta}+u=-\frac{d \phi}{d \zeta} .
\end{aligned}
$$

We also employ simple boundary conditions:

$$
\begin{aligned}
& v=\Psi=0, \quad n=\bar{n} \text { at } z=0, \\
& v \leq c_{s} \quad \text { (Bohm sheath criterion). }
\end{aligned}
$$

In this section, unlike the convention used in previous sections, $z=0$ represents the center of the glow discharge (not the position of the particle).

It is straightforward to show that Eqs. (39)-(41) are equivalent to

$$
\begin{aligned}
& \frac{d \zeta}{d u}=\frac{1-u^{2}}{1+u^{2}}, \\
& \frac{d \phi}{d u}=-2 \frac{u}{1+u^{2}}
\end{aligned}
$$

on $0 \leq u \leq 1$, with $n=\bar{n} \exp \phi$. The solutions to these equations may be obtained easily as

$$
\begin{aligned}
& \zeta=2 \arctan u-u, \\
& \phi=-\ln \left(1+u^{2}\right) .
\end{aligned}
$$

Figures 3 and 4 depict the profiles of density $n$, velocity $v$, field potential $\Psi$, and electric field $E$ in the presheath region given by Eqs. (42) and (43). Here $L_{0}=(\pi / 2-1) c_{s} / v_{\text {ion }}$, and $z=L_{0}$ is the boundary between the presheath and electrode sheath (i.e., dark space) in the discharge.

Using the density and electric field profiles in Figs. 3 and 4 for $n_{i 0}=n_{e 0}$ and $E_{0}$ in Eq. (16), where

$$
k_{D}^{2}(z)=\left(\frac{q^{2} \beta_{i}}{\varepsilon_{0}}+\frac{e^{2} \beta_{e}}{\varepsilon_{0}}\right) n(z),
$$

we may write the $z$ components of the external-field force and polarization force as

$$
\begin{aligned}
& F_{0}(z)=-Q E_{0}(z)=\frac{Q v_{\text {ion }}}{e \beta_{e} c_{s}} f_{0}\left(\frac{z}{L_{0}}\right), \\
& F_{1}(z)=\frac{\sigma(z) Q^{2}}{16 \pi \varepsilon_{0} k_{D}(z)}=\delta \frac{Q v_{\text {ion }}}{e \beta_{e} c_{s}} f_{1}\left(\frac{z}{L_{0}}\right),
\end{aligned}
$$

where $\sigma(z)=d k_{D}(z)^{2} / d z$ and

$$
\delta=\frac{e Q \beta_{e}}{16 \pi \varepsilon_{0} \bar{\lambda}_{D}},
$$




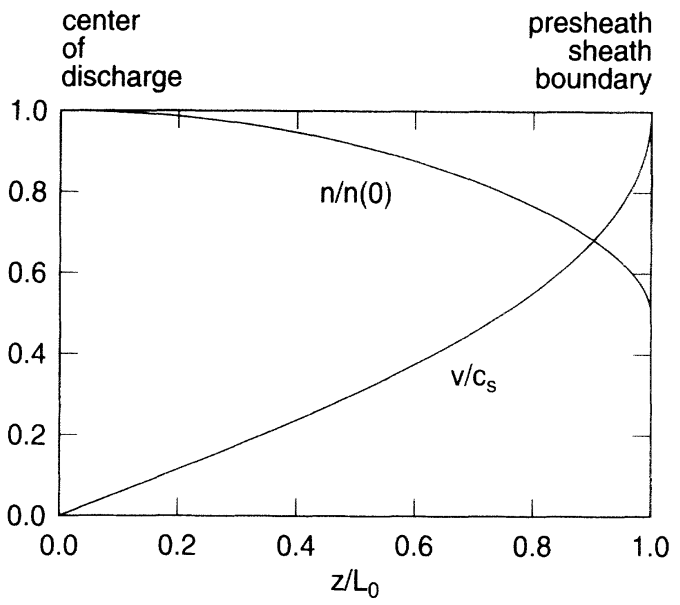

FIG. 3. Profiles of density $n$ and flow velocity $v$ in the presheath region of a glow discharge obtained from Eqs. (42) and (43). The density is normalized by that at $Z=0$, i.e., $\bar{n}=n(0)$, and the flow velocity by the sound speed $c_{s}$. Here $z=0$ is the center of the discharge and $z=L_{0}$ is the interface between the presheath and cathode sheath.

with $\bar{\lambda}_{D}$ being the Debye length at the center of the discharge (i.e., $z=0)$. Clearly $f_{0}\left(z / L_{0}\right)=d \phi / d \xi$ and $f_{1}\left(z / L_{0}\right)=\exp (\phi / 2) d \phi / d \zeta$, where $\phi$ is evaluated as a function of $\zeta=(\pi / 2-1) z / L_{0}$ from Eqs. (42) and (43).

Figure 5 shows $f_{0}$ (i.e., the force $F_{0}$ in the unit of $Q v_{\text {ion }} / e \beta_{e} c_{s}$ ) and $f_{1}$ (i.e., the polarization force $F_{1}$ in the unit of $\delta Q v_{\text {ion }} / e \beta_{e} c_{s}$ as functions of position in the presheath, as calculated from Eqs. (42) and (43). Note that the ratio of the polarization force $F_{1}$ to the external-field force $F_{0}$ is of order $\delta$, i.e.,

$$
\frac{F_{1}}{F_{0}} \simeq \delta
$$

in the presheath. For a particulate of charge $Q=1000 e$ in a plasma with $T_{i}=0.1 \mathrm{eV}, T_{e}=1 \mathrm{eV}$, and $n=1 \times 10^{12}$ $\mathrm{cm}^{-3}$, this ratio becomes $\delta=0.16$ : the magnitude of the sheath polarization force $F_{1}$ is a fraction of the externalfield force $F_{0}=-Q E_{0}$.

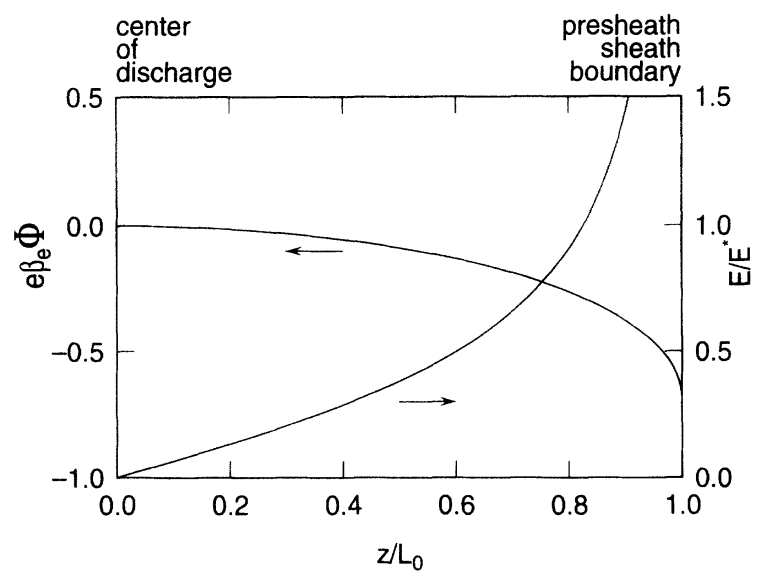

FIG. 4. Profiles of the field potential $\Psi$ (normalized by $1 / e \beta_{e}$ ) and electric field $E$ (normalized by $E^{*}=v_{\text {ion }} / e \beta_{e} c_{s}$ ) in the same presheath region as that of Fig. 3 .

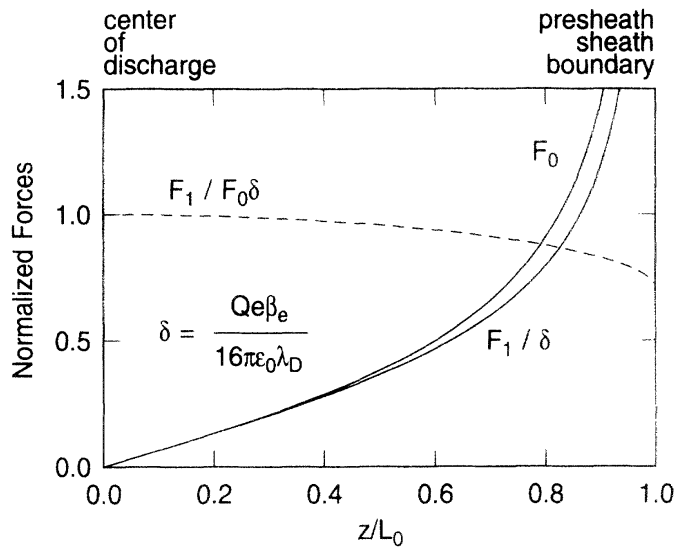

FIG. 5. The external electrostatic force $F_{0}$ and the ratio of the polarization force $F_{1}$ to $\delta$ as functions of position in the presheath region of Figs. 3 and 4. The forces are normalized by $Q v_{\text {ion }} / e \beta_{e} c_{s}$.

We note that the polarization force $F_{1}$ was derived under the assumption that the plasma flow $v$ is negligible, whereas-as shown in Fig. 3-the flow velocity in the presheath region can be large in reality, reaching the ion sound velocity $c_{s}$ at the presheath-sheath boundary $z=L_{0}$. In fact, it is clear from the mass conservation equations (35) and (36) that effects of the velocity gradient may not be negligible:

$$
\frac{1}{n} \frac{d n}{d z} \sim \frac{1}{v} \frac{d v}{d z} .
$$

In this section, therefore, we have simply estimated the magnitude of the force given by Eq. (16), without demonstrating that this is in fact one of the dominant forces exerted on the particulate in the presheath. Indeed, the finite ion flow velocity is known to exert a significant drag force in the direction of the flow. However, aside from this drag force, a more detailed study including the plasma flow effects shows that the force is well approximated by Eq. (16) even in the presence of a finite ion flow.

\section{DISCUSSION}

We have shown that, if plasma flow effects are negligible, the total force on a particulate is given by the sum of the direct electrostatic force exerted by the external field and the sheath-polarization force. For an infinitesimal particle, we obtained the expression for the total force given by Eq. (16), i.e.,

$$
\mathbf{F}=-Q \mathbf{E}_{0}-\frac{Q^{2} \nabla \lambda_{D}}{8 \pi \varepsilon_{0} \lambda_{D}^{2}},
$$

where $\mathbf{E}_{0}$ and $\lambda_{D}$ are evaluated at the particulate position. For a particle of small radius $a$, the force is given by Eq. (33). As seen in both Eqs. (16) and (33), the total force on the charge $-Q$ is given exactly by $-Q \mathbf{E}_{0}$ in the case of uniform density (i.e., $\sigma=0$ ). In other words, as one might expect, an unpolarized, perfectly spherical sheath does not mitigate the externally applied field at 
the particulate position. Only the polarization of the sheath modifies the external field-reducing or enhancing it, depending on the direction of the polarization force relative to that of the applied field. The polarization force is always in the direction of decreasing Debye length, regardless of the sign of the particulate charge or the direction of the applied field. In other words, the free energy of the system-given by Eqs. (17) and (34) - is smaller when the particulate has a thinner Debye sheath.

The presence of finite plasma flow may change the polarization force given by Eq. (44), beyond the obvious effect of a drag force on the particulate. We have also calculated the polarization force in the presence of a finite plasma flow; the results are beyond our present scope and will be presented in due course. For the present, it suffices to note that this study shows that Eq. (44) is correct to $O\left(v_{i 0}^{2} / v_{\text {th }}^{2}\right)$, where $v_{i 0}$ and $v_{\text {th }}$ denote the ion flow velocity and ion thermal velocity $v_{\mathrm{th}}=\left(k_{B} T_{i} / m_{i}\right)^{1 / 2}$.

It can also be shown that, in the presence of a large ion flow velocity (e.g., when $v_{i} \simeq c_{2}=\left(k_{B} T_{e} / m_{i}\right)^{1 / 2}$, the ion sound velocity or Bohm velocity, near the presheathsheath boundary given by $z=L_{0}$ in Sec. VII) the electrostatic and polarization forces are given by Eq. (44) except that one uses the electron Debye length:

$$
\mathbf{F}=-Q \mathbf{E}_{0}-\frac{Q^{2} \nabla \lambda_{e}}{8 \pi \varepsilon_{0} \lambda_{e}^{2}} .
$$

The physical reason for this is that, when the ion flow is sufficiently large compared to the ion thermal velocity (i.e., $v_{i 0} \gg v_{\text {th }}$ ), ions cannot form a sheath, and the sheath comprises a deficiency of electrons. In this case, ions are "blown away" by the fast ion mean flow, and the ion density profile around the particulate becomes almost uniform. As is obvious from the discussion in Sec. II, the constant ion density distribution [instead of Eq. (4)] results in the background Debye length given by the electron Debye length (i.e., $\lambda_{D}=\lambda_{e}$ ). In other words, the thickness of the sheath around a particulate is given by the electron Debye length $\lambda_{e}$ in the presence of a large ion flow.

The total force on a single particulate is thus given by the sum of $F$ above [either by Eqs. (44) or (45) depending on whether the ion flow is small or large] and the ion drag force, i.e., the force due to Coulomb collisions between the flowing ions and the particulate. A complete expression for, and detailed discussion of, this drag force (as well as convenient approximate expressions) may be found in [12].

\section{APPENDIX A: THE ION DENSITY DISTRIBUTION}

In this appendix we further discuss why the Boltzmann ion distribution in Eq. (4) may be used even in regimes beyond its nominal physical validity. We also derive the appropriate "ion Debye length" from a more general ion distribution. To this end, it suffices to assume spherical symmetry around the particulate and a uniform plasma (i.e., $\Psi_{0}=0$ and $\nabla n_{i 0}=\nabla n_{e 0}=0$ ).
As the most general case, we assume that the ion density distribution is given by the form

$$
n_{i}(r)=N_{i}(r, \varphi(r)),
$$

where $r=|\mathbf{r}|$ is the radial distance from the particulate, located at $\mathbf{r}=0$, and $\varphi$ is the potential generated by the particulate. Since $\varphi \rightarrow 0$ as $r \rightarrow \infty$, for large $r$ we may write

$$
\left.n_{i} \simeq \frac{\partial N_{i}}{\partial \varphi}\right|_{r=\infty} \varphi,
$$

which, together with the linearized electron Boltzmann distribution [Eq. (5)], leads to the linearized Poisson's equation

$$
\Delta \varphi^{(\operatorname{lin})}(r)=\frac{Q}{\varepsilon_{0}} \delta(\mathbf{r})+\frac{1}{\lambda_{D}^{2}} \varphi^{(\operatorname{lin})}(\boldsymbol{r})
$$

with

$$
\frac{1}{\lambda_{D}^{2}}=-\left.\frac{q}{\varepsilon_{0}} \frac{\partial N_{i}}{\partial \varphi}\right|_{r=\infty}+\frac{e^{2} n_{e 0}}{k_{B} T_{e}} .
$$

Since the ion density should decrease monotonically to 0 as $r \rightarrow \infty$, we may assume $\partial N_{i} / \partial \varphi<0$ (since $\varphi<0$ ) and define the ion Debye length $\lambda_{i}$ as

$$
\frac{1}{\lambda_{i}^{2}}=-\left.\frac{q}{\varepsilon_{0}} \frac{\partial N_{i}}{\partial r}\right|_{r=\infty}
$$

Poisson's equation (A1) gives the approximate solution $\varphi^{(\text {lin) }}$ for the profile of the potential $\varphi$ appropriate to large $r$. As in the case of Eq. (10), the solution of Eq. (A1) is given by the screened Coulomb potential

$$
\varphi^{(\operatorname{lin})}(r)=-\frac{Q \exp \left(-r / \lambda_{D}\right)}{4 \pi \varepsilon_{0} r} .
$$

To obtain the solution for small $r$, we must solve the full Poisson equation

$$
-\varepsilon_{0} \Delta \varphi(r)=-Q \delta(\mathbf{r})+q n_{i}(r)-e n_{e}(r) .
$$

Suppose the asymptotic form of the solution near $r=0$ is given by $\varphi \sim r^{\alpha}(\alpha<0)$. Noting that $n_{e} \rightarrow 0$ exponentially as $r \rightarrow 0$ and $\Delta=\partial^{2} / \partial r^{2}+(2 / r) \partial / \partial r$, Eq. (A3), near $r=0$, becomes

$$
\alpha(\alpha+1) r^{\alpha-2} \sim q n_{i}(r) .
$$

Thus, if $r^{2-\alpha} n_{i}(r) \rightarrow 0$ as $r \rightarrow 0$, the only possible singularity under these assumptions is $\alpha=-1$, i.e., $\varphi \sim 1 / r$. This choice of $\alpha$ satisfies the condition $r^{2-\alpha} n_{i}(r)=r^{3} n_{i}(r) \rightarrow 0$ : the ion density should not increase any faster than $1 / r^{3}$ near $r=0$.

Therefore, the physical solution $\varphi$ that we are concerned with - the magnitude of which increases monotonically as $r$ decreases from $\infty$ to 0 -is given by $\varphi \simeq \varphi^{\text {(lin) }}$ for large $r$ and $\varphi \sim 1 / r$ for small $r$. For intermediate $r$, the monotonicity requirement is likely to determine the overall functional dependence of $\varphi$ on $r$. Hence it is natural to assume that the qualitative behavior of the full solution $\varphi$ is described reasonably 
well by its linearized form $\varphi^{(\text {lin) }}$, and is relatively insensitive to the exact form of the ion density distribution $n_{i}(r)$.

It is interesting to also note that, as mentioned above, the ion density $n_{i}(r)$ should not increase any faster than $1 / r^{3}$ around $r=0$ in order for the solution $\varphi(r)$ to have a "reasonable" singularity of the form $r^{\alpha}(\alpha<0)$. This implies that the ion Boltzmann distribution given by Eq. (4) may be invalid near $r=0$ not only physically but also mathematically, and should only be used in its linearized form (or, more precisely, including terms to order $\varphi^{2}$ in its Taylor expansion), as was done in Sec. II.

By way of example, consider the collisionless ion density distribution given by Bernstein and Rabinowitz [22]:

$$
\begin{array}{r}
n_{i}(r)=\frac{n_{i 0}}{2}\left[\left(1-\frac{q \varphi}{E_{0}}\right]^{1 / 2} \pm\left(1-\frac{q \varphi}{E_{0}}-\frac{C}{r^{2}}\right)^{1 / 2}\right] \\
\text { for } r \gtrless r_{0},
\end{array}
$$

where $C$ is a constant proportional to the total ion flow to the particulate, and the positive and negative signs should be taken for $r>r_{0}$ and $r<r_{0}$, respectively. Here $r_{0}$ is defined as the self-consistent solution to the equations

$$
\begin{aligned}
& 1-\frac{q \varphi\left(r_{0}\right)}{E_{0}}-\frac{C}{r_{0}^{2}}=0, \\
& \left.r_{0}^{3} \frac{d \varphi}{d r}\right|_{r=r_{0}}=\frac{C E_{0}}{q},
\end{aligned}
$$

which must be solved simultaneously with Poisson's equation to determine $\varphi$. The ion distribution (A4) represents the solution to the collisionless ion Vlasov equation, whose boundary condition is a monoenergetic ion distribution (of energy $E_{0}$ ) outside the sheath. This ion distribution may be regarded as another extreme of simplification - the opposite limit of the collisional case given by the ion Boltzmann distribution (4).

For large $r$, the ion density distribution (A4) may be linearized as

$$
n_{i}(r) \simeq n_{i 0}\left(1-\frac{q \varphi}{2 E_{0}}-\frac{C}{2 r^{2}}\right) .
$$

In the region where $C / 2 r^{2}<q \varphi(r) / E_{0} \ll 1$, therefore, we obtain Poisson's equation (A1) with

$$
\frac{1}{\lambda_{D}^{2}}=\frac{q^{2} n_{i 0}}{2 E_{0}}+\frac{e^{2} n_{e 0}}{k_{B} T_{e}},
$$

and the ion Debye length may be defined as $\lambda_{i}=\sqrt{2 E_{0} / q^{2} n_{i 0}}$. By setting $E_{0}=\frac{1}{2} k_{B} T_{i}$, the characteristic Debye length $\lambda_{D}$ becomes $\lambda_{0}$ used in Sec. III. [Note that, as discussed in [22], the asymptotic form of the potential $\varphi$ for the ion distribution (A4) at large $r$ is given by $\varphi \sim 1 / r^{2}$, rather than the screened Coulomb potential, since the term $C / 2 r^{2}$ in Eq. (A5) may not be ignored for $r \rightarrow \infty$.] Recent numerical calculations [25] also indicate that the screened Coulomb potential with the Debye length (A6) is an excellent approximation of the potential $\varphi(r)$ obtained from the ion distribution (A4).

\section{APPENDIX B: DERIVATION OF EQ. (23)}

Here we elaborate on the derivation of the first-order potential $\varphi^{(1)}(\mathbf{r})$, given by Eq. (25), from Poisson's equation (11) for a finite-size particle. A similar approach may be used to derive the corresponding expression (13) for an infinitesimal particle. In order to make the method applicable to higher-order corrections [i.e., contributions from $O\left(z^{2}\right)$ or higher-order terms in $\Psi_{0}(z)$ and $\left.k_{D}(z)\right]$, we shall generalize the method of derivation and discuss it in the context of a general harmonic expansion of the potential $\varphi(\mathbf{r})$.

Assuming the particle is located at the origin, we take polar coordinates about the $z$ axis. In dimensionless form, Eq. (11) may be written as

$$
\left(\Delta_{\xi}-1\right) \phi_{1}(\xi)=\mu \phi_{0}(\xi) \xi \cos \theta,
$$

where $\xi=k_{0} \mathbf{r}, \xi=|\xi|$, and $\mu$ is the parameter $\sigma / k_{0}^{3}$. The potentials are defined by $\phi_{1}(\xi)=\varphi^{(1)}(\mathbf{r})$ and $\phi_{0}(\xi)=\varphi^{(0)}(r)$ [see Eq. (22)], and $\Delta_{\xi}$ denotes the Laplacian in $\xi$.

Suppose $\phi_{1}(\xi)$ has the form

$$
\phi_{1}(\xi)=g_{l}(\xi) P_{l}(\cos \theta),
$$

where $P_{l}(\zeta)$ is the $l$ th-order Legendre polynomial in $\zeta$. This form comes from the spherical harmonic expansion of the potential $\varphi(\mathbf{r})$. Writing

$$
g_{l}(\xi)=w_{l}(\xi) \xi^{-1 / 2},
$$

the left-hand side of Eq. (B1) becomes

$$
\begin{aligned}
\frac{1}{\xi^{3 / 2}}\left\{\frac{d}{d \xi}\left[\xi \frac{d w_{l}}{d \xi}\right]\right. \\
\left.-\xi\left[1+\frac{\left(l+\frac{1}{2}\right)^{2}}{\xi^{2}}\right] w_{l}\right\} P_{l}(\cos \theta) .
\end{aligned}
$$

Since the right-hand side of Eq. (B1) may be written as $\mu \phi_{0}(\xi) \xi P_{1}(\cos \theta)$, we choose $l=1$ for Eq. (B2). Note that for higher-order calculations, where the left-hand side is proportional to a general $P_{l}(\cos \theta)$, the corresponding in$\operatorname{dex} l$ has to be chosen for Eq. (B2).

To solve the resulting equation, we use the Green's function $G_{v}(x, y)$ for the modified Bessel differential equation, defined by

$$
\frac{d}{d x}\left(x \frac{d G_{v}}{d x}\right)-x\left(1+\frac{v^{2}}{x}\right) G_{v}=-\delta(x-y),
$$

where $x>0$. This Green's function can easily be constructed as

$$
G_{v}(x, y)= \begin{cases}K_{v}(y) I_{v}(x) & \text { for } x<y \\ K_{v}(x) I_{v}(y) & \text { for } x>y,\end{cases}
$$

where $K_{v}$ and $I_{v}$ are the $v$ th-order modified Bessel functions. In our case, we choose $v=\frac{3}{2}$, i.e.,

$$
K_{3 / 2}(z)=\left(\frac{\pi}{2 z}\right)^{1 / 2}\left(1+\frac{1}{z}\right) \exp (-z),
$$




$$
I_{3 / 2}(z)=\left(\frac{2}{\pi z}\right]^{1 / 2}\left[\cosh z-\frac{1}{z} \sinh z\right] .
$$

Then a special solution $\phi_{1}^{s}(x)=g_{1}^{s}(\xi) \cos \theta$ to Eq. (B1) may be obtained as

$$
\begin{aligned}
g_{1}^{s}(\xi) & =w_{1}(\xi) \xi^{-1 / 2} \\
& =-\frac{\mu}{\sqrt{\xi}} \int_{0}^{\infty} \xi^{5 / 2} \phi_{0}(\xi) G_{3 / 2}(\xi, y) d y \\
& =-\frac{1}{4} \mu C \exp (-\xi) \xi,
\end{aligned}
$$

where we set $\phi_{0}(\xi)=C \exp (-\xi) / \xi \quad$ with $C=k_{0} a V_{s} \exp \left(k_{0} a\right)$. Using arbitrary constants $C_{1}$ and
$C_{2}$ and the special solution $g_{1}^{s}$ above, we obtain the general solution to Eq. (B1) as $\phi_{1}(x)=g_{1}(\xi) \cos \theta$, where

$g_{1}(\xi)=C_{1} I_{3 / 2}(\xi) \xi^{-1 / 2}+C_{2} K_{3 / 2}(\xi) \xi^{-1 / 2}+g_{1}^{s}(\xi)$.

From the boundary condition (18), we have $g_{1}(\xi) \rightarrow 0$ as $\xi \rightarrow \infty$, which leads to $C_{1}=0$. Applying the boundary condition (19) to the mean field $\Psi_{0}(z)=\Psi_{0}(0)-E_{0} r \cos \theta$, and the perturbed field $\varphi(\mathbf{r})=\varphi^{(0)}(r)+g_{1}\left(k_{0} r\right) \cos \theta$, we obtain

$$
-a E_{0}+g_{1}\left(k_{0} a\right)=0 .
$$

This boundary condition determines the constant $C_{2}$ and thus yields Eq. (25).
[1] B. Ganguly, A. Garscadden, J. Williams, and P. Haaland, J. Vac. Sci. Technol. A 11, 1119 (1993).

[2] R. N. Carlile, S. Geha, J. F. O'Hanlon, and J. C. Stewart, Appl. Phys. Lett. 59, 1167 (1991).

[3] A. Bouchoule, A. Plain, L. Boufendi, J. Ph. Blondeau, and C. Laure, J. Appl. Phys. 70, 1991 (1991).

[4] G. S. Selwyn, J. Singh, and R. S. Bennett, J. Vac. Sci. Technol. A 7, 2758 (1989).

[5] K. G. Spears, T. J. Robinson, and R. M. Roth, IEEE Trans. Plasma Sci. 14, 179 (1986).

[6] H. Ikezi, Phys. Fluids 29, 1764 (1986).

[7] R. T. Farouki and S. Hamaguchi, Appl. Phys. Lett. 61, 2973 (1992).

[8] R. T. Farouki and S. Hamaguchi, Phys. Rev. E 47, 4330 (1993).

[9] Strongly Coupled Plasma Physics, edited by F. J. Rogers and H. E. DeWitt (Plenum, New York, 1986).

[10] M. Baus and J.-P. Hansen, Phys. Rep. 59, 1 (1980).

[11] S. G. Brush, H. L. Sahlin, and E. Teller, J. Chem. Phys. 45, 2102 (1966).

[12] T. G. Northrop and T. J. Birmingham, Planet. Space Sci. 38, 319 (1990).
[13] B. A. Trubnikov, in Review of Plasma Physics, edited by M. A. Leontovich (Consultants Bureau, New York, 1965), Vol. 1, p. 205.

[14] S. Chandrasekahar, Principles of Stellar Dynamics (Dover, New York, 1960).

[15] L. Spitzer, Jr., Physics of Fully Ionized Gases, 2nd ed. (Interscience, New York, 1962).

[16] N. A. Krall and A. W. Trivelpiece, Principles of Plasma Physics (McGraw-Hill, New York, 1973).

[17] J. Hubbard, Proc. R. Soc. London Ser. A 260, 114 (1961).

[18] T. Kihara and O. Aono, J. Phys. Soc. Jpn. 18, 837 (1963).

[19] See, for example, D. H. Everett, Basic Principles of Colloid Science (The Royal Society of Chemistry, London, 1988).

[20] T. G. Northrop, Phys. Scr. 45, 475 (1992).

[21] E. C. Whipple, T. G. Northrop, and D. A. Mendis, J. Geophys. Res. 90, 7405 (1985).

[22] I. Bernstein and I. Rabinowitz, Phys. Fluids 2, 112 (1959).

[23] S. J. Choi and M. J. Kushner, Appl. Phys. Lett. 62, 2197 (1993).

[24] J. P. Boeuf, Phys. Rev. A 46, 7910 (1992).

[25] J. E. Daugherty, R. K. Porteous, M. D. Kilgore, and D. B. Graves, J. Appl. Phys. 72, 3934 (1992). 